\title{
Layered composite and contact layer. Effective modulus of elasticity
}

\author{
Vladimir Andreev, Nikita Tsybin* and Robert Turusov \\ Moscow State University of Civil Engineering, Yaroslavskoe shosse, 26, Moscow, 129337, Russia
}

\begin{abstract}
The effective modulus of elasticity of a layered composite is one of the important characteristics. At the moment, the so-called mixture formula is most often used to calculate this characteristic. However, as a number of experiments demonstrate, the formula of the mixture does not always allow calculating the effective modulus of elasticity. The reason for this fact is the inhomogeneous stress-strain state arising in a layered composite. This article presents a method for finding the effective modulus of elasticity of a layered composite based on the contact layer model. Such approach to the calculation allows us to take into account not only the volume content of the composite components, but also many other parameters of the model.
\end{abstract}

\section{Statement of the problem}

The model of layered composite shown in Fig. 1 is considered. In the article [1] the system

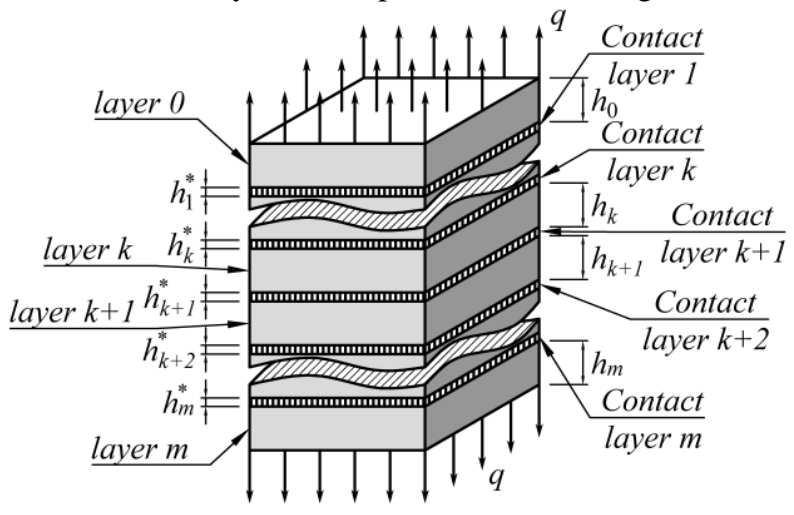

Fig 1. Model of layered composite of resolving equations for this model was obtained. The main objective of this article is to find the effective modulus of elasticity of a layered composite. In this regard, the focus is on the normal stresses arising in layers of adhesive and substrate. The article [1] shows that the difference in normal stresses at using a simplified model and a contact layer model of a general form is insignificant. The main difference of these models is the distribution of the shear stresses near the side faces of the composite. This allows us to use here a simplified model of the contact layer. We present the obtained system of resolving equations without changes

* Corresponding author: science@nikitatsybin.ru 


$$
\left.\begin{array}{l}
h_{k} \frac{\partial^{2} \sigma_{x, k}}{\partial x^{2}}=-\frac{G^{*}}{h^{*}}\left(\varepsilon_{x, k-1}-2 \varepsilon_{x, k}+\varepsilon_{x, k+1}\right) \\
h_{k} \frac{\partial^{2} \sigma_{x, k}}{\partial y^{2}}=-\frac{G^{*}}{h^{*}}\left(\varepsilon_{y, k-1}-2 \varepsilon_{y, k}+\varepsilon_{y, k+1}\right) .
\end{array}\right\}
$$

in which

$$
\left.\begin{array}{l}
\varepsilon_{x, k}=\frac{1}{E_{k}}\left[\sigma_{x, k}-v_{k}\left(q+\sigma_{y, k}\right)\right] ; \\
\varepsilon_{y, k}=\frac{1}{E_{k}}\left[\sigma_{y, k}-v_{k}\left(q+\sigma_{x, k}\right)\right] ; \\
\varepsilon_{z, k}=\frac{1}{E_{k}}\left[q-v_{k}\left(\sigma_{x, k}+\sigma_{y, k}\right)\right] .
\end{array}\right\}
$$

For clarity, we present the expressions from which the shear stresses are determined

$$
\tau_{x z, k}^{*}-\tau_{x z, k+1}^{*}=h_{k} \frac{\partial \sigma_{x, k}}{\partial x} ; \quad \tau_{y z, k}^{*}-\tau_{y z, k+1}^{*}=h_{k} \frac{\partial \sigma_{y, k}}{\partial y} .
$$

This system of equations makes it possible to determine the components of the stressstrain state of a layered composite with any number of layers.

Let us denote the hypotheses that were used in the derivation of the resolving equations for the problem:

1) The adhesive and substrate layers are isotropic and obey Hooke's law.

2) The interaction of the adhesive and substrate layers is implemented through a contact layer.

3) The contact layer is considered as an anisotropic elastic medium. The parameters of this medium are such that it can be represented as a thick brush of elastic rods, not connected to each other and oriented normally to the contact surface. In such a medium, there are no normal stresses in the contact plane (perpendicular to the "lateral" surface of the rods), and the Poisson's ratio (since the rods are not connected) is zero. As a result, the main characteristics of the contact layer to be determined from the macro experiment are its thickness $h^{*}$ and shear stiffness $G^{*}$, and since the Poisson's ratio is assumed to be zero, the shear modulus is associated with the Young's modulus by a simple equation $G^{*}=E^{*} / 2$. The basic relations of the theory of elasticity of anisotropic bodies (equilibrium equations, Hooke's law and Cauchy relations) remain unchanged.

4) The loading scheme of the model assumes that the lateral faces are free from loads. A tensile stress $q$ is applied to the top and bottom faces.

5) It is assumed that the stiffness of the bonded substrate layers is such that the bending can be neglected. As a result, the stresses $\sigma_{z}$ are constant throughout the model and equal to the value of the applied tensile load, i.e. $\sigma_{z}=q$.

6) Since there is no bending, deformations $\varepsilon_{x}$ and $\varepsilon_{y}$ of layers of adhesive and substrate, displacements $u$ and $v$, as well as stresses $\sigma_{x}$ and $\sigma_{y}$ are functions of two variables $x$ and $y$.

7) It is assumed that there are only elastic deformations in each layer $k$ of the composite.

\section{Experiment}

Let us consider the following experiment, discussed in detail in [2]. 
Three rods are made at the same time. The first rod consists of three series-connected rods - steel - epoxy - steel. The length of the steel rods is $42.5 \mathrm{~mm}$, the length of the epoxy

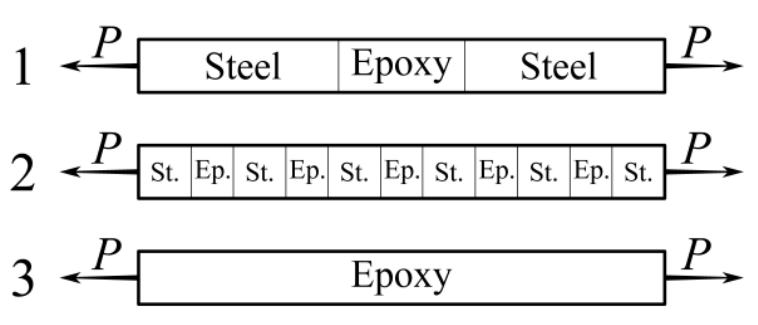

Fig 2. Models from the experiment

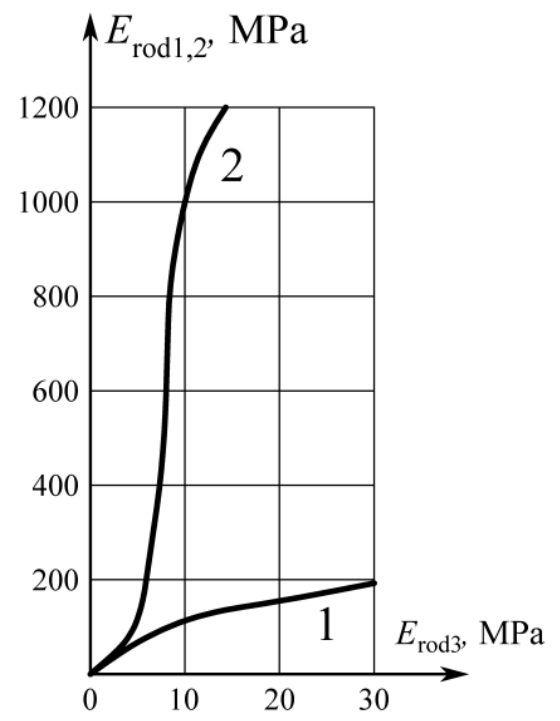

Fig 3. Results of the experiment

rod located between them is $15 \mathrm{~mm}$. The cross sections of the rods are the same $10 \times 10 \mathrm{~mm}$. The second rod is a multilayer structure (the layers are perpendicular to the longitudinal axis) of alternating layers of steel with a thickness of $1.4 \mathrm{~mm}$ and layers of epoxy polymer with a thickness of $0.25 \mathrm{~mm}$. The second rod has the same relative proportions of polymer and steel as the first rod. The third rod is completely made of epoxy resin.

All three rods use liquid epoxide with a hardener. A graphic illustration of the three rods considered is shown in Fig. 2.

As the epoxy polymer hardens, Young's modulus (by the quasi-static method) is measured for all three rods at low loads.

Fig. 3 presents the results of measuring the Young's modulus of the composite (rod 1, curve 1) and a layered rod (rod 2, curve 2) depending on the Young's modulus of the polymer. The Young's modulus of the polymer was measured at the same time points on a rod of pure epoxide ( $\operatorname{rod} 3)$.

Fig. 3 demonstrates a significantly different course of curves 1 and 2 . Initially, at small values of the Young's modulus of the polymer, the results obtained on both rods coincide and then begin to diverge. Young's modulus with thin polymer layers begins to increase sharply (curve 2), many times exceeding the modulus of the three-section rod (curve 1). In this case, the course of curve 1 for a three-section rod is well described by the known mixture formula for the Young's modulus across the layers.

It is obvious that the reasons for such a difference should be sought in a significantly inhomogeneous stress-strain state arising in the layered composite. 


\section{Three-layer model}

Let us consider a three-layer composite consisting of two identical layers of substrate,

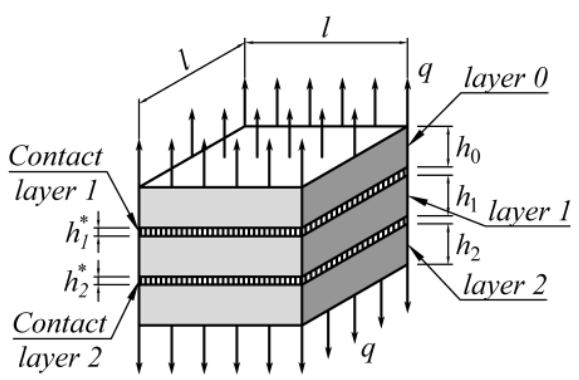

Fig. 4. Model with three layers bonded with adhesive (Figure 3). For simplicity, the section will be considered square with dimensions $l \times l$.

Since the cross section is square, in equations (1), (2) and (3) it is necessary to take into account the fact that at points with the same values $x, y$ the stresses $\sigma_{x, k}$ and $\sigma_{y, k}$ are equal.

Due to the fact that the layers of the substrate are the same, we assume:

$E_{0}=E_{2}=E_{s} ; v_{0}=v_{2}=v_{s} ; h_{0}=h_{2}=h_{s} ; h_{1}=h_{a} ; E_{1}=E_{a} ; v_{1}=v_{a}$.

From the equilibrium condition of the composite as a whole, we obtain the following relations:

$$
\sigma_{x, 0} h_{0}=\sigma_{x, 2} h_{2}=-\frac{1}{2} \sigma_{x, 1} h_{1} \rightarrow \sigma_{x, 0}=\sigma_{x, 2}=-\frac{\sigma_{x, 1} h_{a}}{2 h_{s}} ; \tau_{x z, 1}^{*}=-\tau_{x z, 2}^{*}=\tau^{*} .
$$

From equality (3), taking into account the last expression (5), we find the shear stresses in the contact layer.

$$
\tau_{x z, 1}^{*}-\tau_{x z, 2}^{*}=2 \tau^{*}=h_{1} \frac{d \sigma_{x, 1}}{d x} \rightarrow \tau^{*}=\frac{h_{a}}{2} \frac{d \sigma_{x, 1}}{d x} .
$$

As a result, the systems of resolving equations can be reduced to the equations for the stresses $\sigma_{x, 1}$ in the adhesive layer:

in which

$$
\frac{d^{2} \sigma_{x, 1}}{d x^{2}}-\sigma_{x, 1} \omega^{2}+q \lambda_{q}^{2}=0
$$

$$
\omega^{2}=\frac{G^{*}}{h_{a} h^{*}}\left[\frac{2\left(1-v_{a}\right)}{E_{a}}+\frac{h_{a}}{h_{s}} \frac{\left(1-v_{s}\right)}{E_{s}}\right] ; 2 \eta=\frac{12}{\left(h^{*}\right)^{2}} ; \lambda_{q}^{2}=\frac{2 G^{*}}{h_{a} h^{*}}\left(\frac{v_{a}}{E_{a}}-\frac{v_{s}}{E_{s}}\right) .
$$

The general solution of equation (7) is written below.

$$
\sigma_{x, 1}=\frac{q \lambda_{q}^{2}}{\omega^{2}}+C_{1} \exp (\omega x)+C_{2} \exp (-\omega x)
$$

The unknown integration constants included in equation (9), are determined from the boundary conditions written below

$$
\sigma_{x, 1}\left( \pm \frac{l}{2}\right)=0
$$

Using the boundary conditions (10), we find

$$
\sigma_{x, 1}=\frac{q \lambda_{q}^{2}}{\omega^{2}}\left[1-\frac{\cosh (\omega x)}{\cosh (\omega l / 2)}\right] \text {. }
$$

The expression (11) allows us to define deformations arising in a three-layer composite. layer:

From equality (6) using the expressions (11) we find the shear stresses in the contact 


$$
\tau^{*}=-\frac{h_{a}}{2} \frac{q \lambda_{q}^{2}}{\omega} \frac{\sinh (\omega x)}{\cosh (\omega l / 2)}
$$

Deformations in the direction $z$ arising in the free prismatic polymer rod for the case of uniaxial stress-strain state are calculated by the formula:

$$
\varepsilon_{z, r}=\varepsilon_{r o d}=\frac{q}{E_{1}}
$$

For a three-layer composite, the total deformation is determined by the sum of the adhesive and substrate deformations.

$$
\varepsilon_{z, c o m}=\varepsilon_{z, 0} \mu_{0}+\varepsilon_{z, 1} \mu_{1}+\varepsilon_{z, 2} \mu_{2} .
$$

In formula (14) $\mu_{i}$ - volume content of the corresponding layer

$$
\begin{aligned}
\mu_{0(2)}= & \frac{\mu_{s}}{2}=\frac{h_{s}}{2 h_{s}+h_{a}} ; \mu_{1}=\mu_{a}=\frac{h_{a}}{2 h_{s}+h_{a}} ; \\
& \mu_{0}+\mu_{1}+\mu_{2}=\mu_{s}+\mu_{a}=1 .
\end{aligned}
$$

Let us determine the deformations of the layers in the direction $z$ :

$$
\left.\begin{array}{c}
\varepsilon_{z, 1}=\varepsilon_{z, a}=\frac{1}{E_{1}} \cdot\left(q-2 v_{1} \cdot \sigma_{x, 1}\right) ; \\
\varepsilon_{z, 0(2)}=\varepsilon_{z, s}=\frac{1}{E_{0}} \cdot\left(q-2 v_{0} \cdot \sigma_{x, 0(2)}\right)=\frac{1}{E_{0}} \cdot\left(q+2 v_{0} \cdot \frac{\sigma_{x, 1} h_{1}}{2 h_{0}}\right) \cdot
\end{array}\right\}
$$

Since these values vary in the cross-sectional area of the composite, we will consider their average values

$$
\left.\begin{array}{c}
\bar{\varepsilon}_{z, s}=\frac{1}{l^{2}} \cdot \int_{-l / 2}^{l / 2} \int_{-l / 2}^{l / 2} \varepsilon_{z, 0(2)}(x) d x d x=\frac{q}{E_{s}} \frac{h_{a} v_{s}}{h_{s}} \frac{\lambda_{q}^{2}}{\omega^{2}}\left[1-\frac{2}{\omega l} \tanh \left(\frac{\omega l}{2}\right)\right]+\frac{q}{E_{s}} ; \\
\bar{\varepsilon}_{z, a}=\frac{1}{l^{2}} \cdot \int_{-l / 2}^{l / 2} \int_{-l / 2}^{l / 2} \varepsilon_{z, 1}(x) d x d x=\frac{q}{E_{a}} 2 v_{a} \frac{\lambda_{q}^{2}}{\omega^{2}}\left[\frac{2}{\omega l} \tanh \left(\frac{\omega l}{2}\right)-1\right]+\frac{q}{E_{a}} .
\end{array}\right\}
$$

Let us find the value of the total deformation

$$
\bar{\varepsilon}_{z, c o m}=\frac{q}{E_{e f}}=q\left(\frac{\mu_{s}}{E_{s}}+\frac{\mu_{a}}{E_{a}}\right)+q \frac{\lambda_{q}^{2}}{\omega^{2}}\left[\frac{2}{\omega l} \tanh \left(\frac{\omega l}{2}\right)-1\right]\left(\frac{2 E_{0} h_{0} v_{1} \mu_{1}-E_{1} h_{1} v_{0} \mu_{0}}{E_{0} E_{1} h_{0}}\right),
$$

from which we can obtain an expression for the effective modulus of elasticity

$$
E_{e f}=\left\{\left(\frac{\mu_{s}}{E_{s}}+\frac{\mu_{a}}{E_{a}}\right)+\frac{\lambda_{q}^{2}}{\omega^{2}}\left[\frac{2}{\omega l} \tanh \left(\frac{\omega l}{2}\right)-1\right]\left(\frac{2 E_{0} h_{0} v_{1} \mu_{1}-E_{1} h_{1} v_{0} \mu_{0}}{E_{0} E_{1} h_{0}}\right)\right\}^{-1} .
$$

In the limit, when the gluing area tends to zero, the formula (19) is converted to the classical mixture formula.

$$
\lim \left(E_{e f}\right) \stackrel{l=0}{\longrightarrow} E_{m i x}=\left(\frac{\mu_{s}}{E_{s}}+\frac{\mu_{a}}{E_{a}}\right)^{-1}
$$




\section{Results of calculation}

The following parameters are used in the calculation:

$$
\begin{gathered}
h_{s}=10 \mathrm{~mm} ; h_{a}=1 \mathrm{~mm} ; l=20 \mathrm{~mm} ; E_{s}=2 \cdot 10^{5} \mathrm{MPa} \\
E_{a}=2 \cdot 10^{3} \mathrm{MPa} ; v_{s}=0.3 ; v_{a}=0.37 ; \frac{G^{*}}{h^{*}}=10^{4} \mathrm{MPa} / \mathrm{mm}
\end{gathered}
$$

Below are the results of calculating the effective elastic modulus of a layered composite depending on the thickness and elastic modulus of the adhesive.

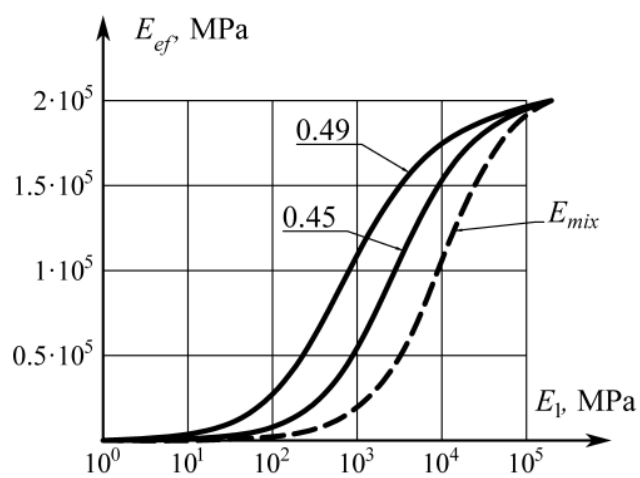

Fig. 5. Dependence of the effective elastic modulus of a layered composite on the elastic modulus of the adhesive

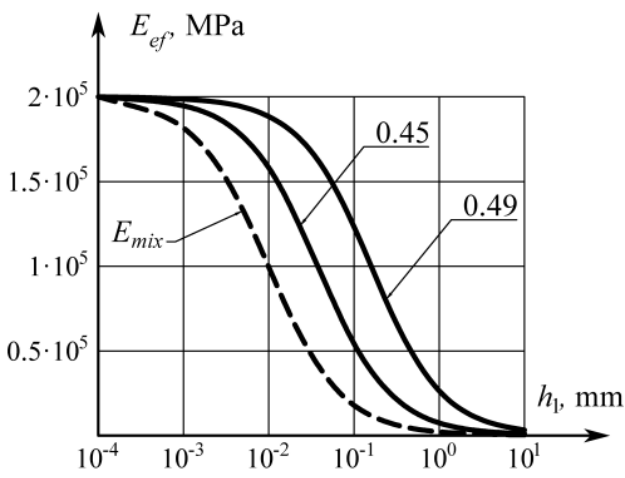

Fig. 6. Dependence of the effective elastic modulus of a layered composite on the thickness of the adhesive layer

In these figures, dashed lines represent the results obtained by the formula of the mixture (20). Solid lines represent the results obtained by the formula (19). The captions for the solid lines correspond to the Poisson's ratio used in the calculation.

The curves obtained coincide only at the limit points. On the intermediate interval the differences are significant. At the same time, the higher the Poisson's ratio, the greater the differences in the results. This is more clearly demonstrated by the graphs of the form $E_{e f} / E_{\text {mix }}$ presented below.

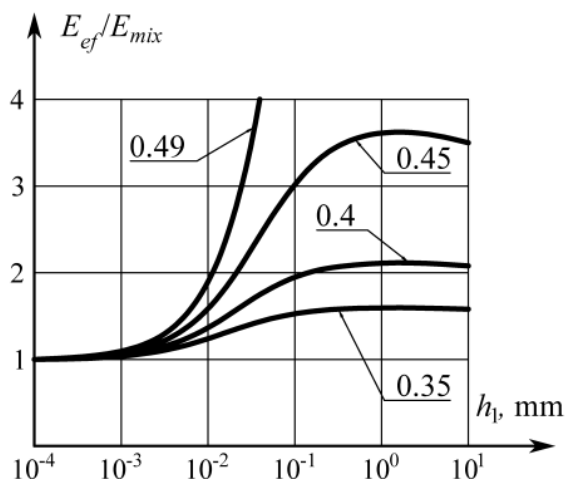

Fig. 7. The ratio between the results obtained by formulae (19) and (20) depending on the thickness of the adhesive layer

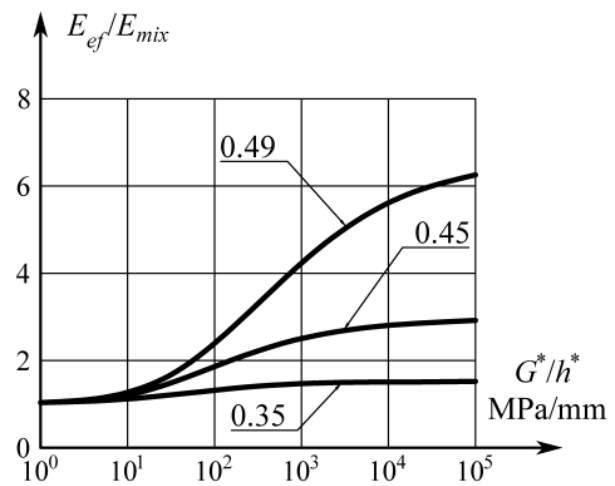

Fig. 8. The ratio between the results obtained by formulae (19) and (20) depending on the intensity of the adhesion interaction

It follows from Fig. 8 that an increase in the stiffness of the contact layer leads to an increase in the effective elastic modulus of the layered composite. This is due to the fact 
that the increase in the stiffness of the contact layer leads to the increase in the normal stresses $\sigma_{x}$ in the adhesive layer, and as a result, it leads to a decrease in deformations $\varepsilon_{z}$.

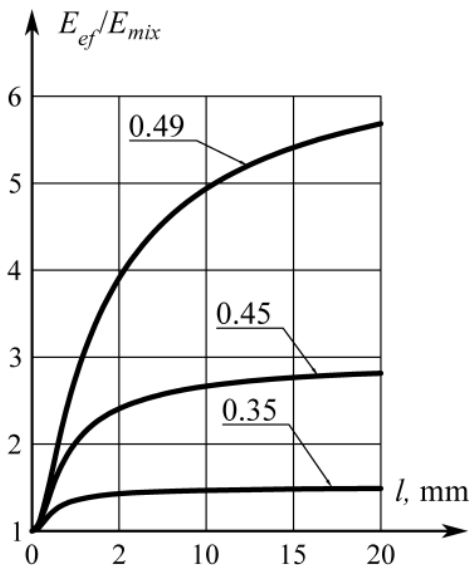

Fig. 9. The ratio between the results obtained by formulae (19) and (20)

depending on the gluing area.

Fig. 9 shows the results of calculating the ratio between the effective elastic modulus of a layered composite obtained by the formula (19) and the formula of the mixture (20).

The gluing area does not affect the result obtained by the formula of the mixture (20). It follows that the increase of curves in Fig. 9 is associated with the increase of the effective modulus of elasticity calculated by the formula (19). The increase of the curves has an asymptotic character, since starting from a certain value, the gluing area ceases to affect the value of normal stresses $\sigma_{x}$. This fact was demonstrated in article [1].

It is also worth noting that with a small gluing area, the calculation results using the formula of the mixture (20) and formula (19) are the same. From this it follows that from the point of view of mechanics, we can not expect synergy from the use of nanoscale

fillers in the composite.

\section{Conclusions}

The calculations showed that the effective modulus of elasticity of the layered composite, in addition to the volume content and Young's modulus of the adhesive and substrate layers, is significantly influenced by the ratio of the adhesive layer thickness to the gluing area, as well as the Poisson's ratio of the adhesive layer. Moreover, the results of the calculation of the effective modulus by the formula of the mixture (20) can be ten times less than the results obtained by the formula (19). It is obvious that such a phenomenon is manifested by the appearance of a significantly inhomogeneous stress-strain state in the model. The results of the experiment, presented at the beginning of the article, are explained (Fig. 3).

This work was financially supported by the Ministry of Education and Science (state task $\#$ 7.1524.2017/4.6).

\section{References}

1. V. Andreev, R. Turusov, N. Tsybin, MATEC Web of Conferences 251, 04066 (2018)

2. R. Turusov, Mechanics of Composite Materials 50(6), 1119-30 (2014)

3. N. Tsybin, R. Turusov, V. Andreev, A. Kolesnikov, MATEC Web of Conferences 196(6), 01057(2018)

4. E. Korol, IOP Conference Series Materials Science and Engineering, 456(1), 012075 (2018)

5. T. Bobyleva, A. Shamaev, MATEC Web of Conferences 251(8), 04039 (2018)

6. E. Kosheleva, MATEC Web of Conferences 117, 00086 (2017)

7. A. Veremeenko, A. Chepurnenko, P. Shvetsov, L. Zorchenko, S. Yazyev, IOP Conference Series Earth and Environmental Science 90(1), 012099 (2017)

8. R. Turusov, Adhesion mechanics (NRU MGSU, 2015) 
9. V. Vasiliev, E. Morozov, Mech. and analysis of composite materials (Elsevier 2001)

10. R. Turusov, A. Kuperman, Polymer Science. Series D, 7(1), 1-8 (2014)

11. K. Kaw, Mechanics of composite materials (Taylor \& Francis 2006) 